\title{
SUBMERSIONS AND IMMERSIONS WITH CODIMENSION ONE OR TWO
}

\section{EMERY THOMAS ${ }^{1}$}

1. Introduction. Let $M$ and $N$ be smooth manifolds and $f: M \rightarrow N$ a smooth map. We say that $f$ has maximal rank if at each point of $M$ the Jacobian matrix of $f$ has maximal rank. If $\operatorname{dim} M<\operatorname{dim} N$, then $f$ is an immersion; while if $\operatorname{dim} M>\operatorname{dim} N, f$ is a submersion. For convenience we define the integer $|\operatorname{dim} M-\operatorname{dim} N|$ to be the codimension of any map $M \rightarrow N$. We consider in this note the following problem. Let $g: M \rightarrow N$ be a continuous map of codimension one or two. When is $g$ homotopic to a smooth map of maximal rank? By exploiting the work of Hirsch [3] and Phillips [6] we obtain answers in terms of cohomology invariants of $M$ and $N$.

All manifolds in the paper will be smooth, connected, and without boundary. For any such manifold $V$ we let $\tau_{V}$ denote the tangent bundle of $V$.

Recall that 1-plane bundles over a complex $X$ are in 1-1 correspondence with $H^{1}\left(X ; Z_{2}\right)$. For each class $u \in H^{1}\left(X ; Z_{2}\right)$ let $\eta(u)$ denote the 1 -plane bundle such that $w_{1}(\eta(u))=u$. (Here for any vector bundle $\xi$ over $X$, we let $w_{i}(\xi) \in H^{i}\left(X ; Z_{2}\right), i \geqq 0$, denote the $i$ th StiefelWhitney class of $\xi$.) Similarly oriented 2-plane bundles over $X$ are in 1-1 correspondence with $H^{2}(X ; Z)$. For each $v \in H^{2}(X ; Z)$, let $\xi(v)$ denote the oriented 2-plane bundle with Euler class $v$.

For a bundle $\xi$ we let $(\xi)$ denote the stable equivalence class determined by $\xi$.

Now let $M$ and $N$ be manifolds and $f: M \rightarrow N$ a continuous map of codimension one or two. We consider separately these two cases.

Case 1: Codimension $f=1$. By the work of Hirsch [3] and Phillips [6] (as reworded in [11, Section 1]), we have at once:

Theorem 1.1. (a) Suppose that $\operatorname{dim} M=\operatorname{dim} N-1$. Then $f$ is homotopic to an immersion iff there is a class $u \in H^{1}\left(M ; Z_{2}\right)$ such that $\left(\tau_{M} \oplus \eta(u)\right)=f^{*}\left(\tau_{N}\right)$. (b) Suppose that $\operatorname{dim} M=\operatorname{dim} N+1$ and that $M$ is open. Then $f$ is homotopic to a submersion iff there is a class $u$ $\in H^{1}\left(M ; Z_{2}\right)$ such that $\left(\tau_{M}\right)=\left(f^{*} \tau_{N} \oplus \eta(u)\right)$.

We will say that a map $f: M \rightarrow N$ is orientable if $f^{*} w_{1} N=w_{1} M$.

Case 2: Codimension $f=2, f$ orientable.

Received by the editors February 14, 1967.

${ }^{1}$ Research supported by the National Science Foundation and the Miller Institute for Basic Research. 
Theorem 1.2. (a) Suppose that $\operatorname{dim} M=\operatorname{dim} N-2$ and that $f: M \rightarrow N$ is an orientable map. Then $f$ is homotopic to an immersion iff there is a class $v \in H^{2}(M ; Z)$ such that $\left(\tau_{M} \oplus \xi(v)\right)=f^{*}\left(\tau_{N}\right)$. (b) Suppose that $\operatorname{dim} M=\operatorname{dim} N+2$, that $M$ is open and that $f: M \rightarrow N$ is orientable. Then $f$ is homotopic to a submersion iff there is a class $v \in H^{2}(M ; Z)$ such that $\left(\tau_{M}\right)=\left(f^{*} \tau_{N} \oplus \xi(v)\right)$.

Again the result follows from Hirsch-Phillips, as given in [11].

The equations given in Theorems 1.1 and 1.2 can be regarded as equations in the $K$-theory cohomology of Atiyah-Hirzebruch [1]. In order to get conditions expressed solely in terms of singular cohomology, we need to restrict drastically the class of manifolds. We will say that a manifold $M$ satisfies Condition $\left({ }^{*}\right)$ if it has the following properties.

Condition $(*)$.

(i) $M \leqq 9$; if $\operatorname{dim} M=9, M$ is open;

(ii) $H^{4}(M ; Z)$ has no 2-torsion;

(iii) $H^{8}(M ; Z)$ has no 6-torsion.

Notice that a closed orientable manifold of $\operatorname{dim} \leqq 4$ satisfies Condition $\left({ }^{*}\right)$, as does a closed orientable manifold of $\operatorname{dim} \leqq 8$ provided (ii) is satisfied.

We combine Theorems 1.1 and 1.2 with [10] to give the following results. The proofs will be given in $\S 3$. (For a bundle $\xi$ over $X$, we denote by $P_{i}(\xi) \in H^{4 i}(X ; Z)$ the $i$ th Pontryagin class of $\xi, i \geqq 0$.)

Theorem 1.3. Let $M$ be a manifold satisfying Condition (*) and let $f: M \rightarrow N$ be a map of codimension 1 . (a) Suppose that $\operatorname{dim} N>\operatorname{dim} M$. Then $f$ is homotopic to an immersion if and only if there is a class $u \in H^{1}\left(M ; Z_{2}\right)$ such that

$$
w_{i}(M)+w_{i-1}(M) \cdot u=f^{*} w_{i}(N), \quad i=1,2,
$$

and

$$
P_{i}(M)=f^{*} P_{i}(N), \quad i=1,2 .
$$

(b) Suppose that $\operatorname{dim} M>\operatorname{dim} N$ and that $M$ is open. Then $f$ is homotopic to a submersion if and only if there is a class $u \in H^{1}\left(M ; Z_{2}\right)$ such that

$$
w_{i}(M)=f^{*} w_{i}(N)+f^{*} w_{i-1}(N) \cdot u, \quad i=1,2,
$$

and

$$
P_{i}(M)=f^{*} P_{i}(N), \quad i=1,2 .
$$

There are similar results for codimension 2 . 
Theorem 1.4. Let $M$ be a manifold satisfying Condition $\left(^{*}\right)$ and let $f: M \rightarrow N$ be an orientable map of codimension 2. (a) Suppose that $\operatorname{dim} M<\operatorname{dim} N$. Then $f$ is homotopic to an immersion if and only if there is a class $v \in H^{2}(M ; Z)$ such that

$$
\begin{aligned}
w_{2}(M)+f^{*} w_{2}(N) & =v \bmod 2, \\
P_{i}(M)+P_{i-1}(M) \cdot v^{2} & =f^{*} P_{i}(N), \quad i=1,2 .
\end{aligned}
$$

(b) Suppose that $\operatorname{dim} M>\operatorname{dim} N$ and that $M$ is open. Then $f$ is homotopic to a submersion if and only if there is a class $v \in H^{2}(M ; Z)$ such that

$$
\begin{gathered}
w_{2}(M)+f^{*} w_{2}(N)=v \bmod 2, \\
P_{i}(M)=f^{*} P_{i}(N)+f^{*} P_{i-1}(N) \cdot v^{2}, \quad i=1,2 .
\end{gathered}
$$

2. Examples. To illustrate the preceding material we take $N$ to be one of the three projective spaces, real, complex, or quaternionic, which we denote respectively by $R P^{n}($ of $\operatorname{dim} n), C P^{n}($ of $\operatorname{dim} 2 n)$, and $Q P^{n}$ (of $\operatorname{dim} 4 n$ ). The determination of all maps of maximal rank from a manifold $M$ into one of these manifolds $N$ falls naturally into two parts. First, the determination of the set of homotopy classes of maps $[M, N]$; and then, the determination of which homotopy classes of maps contain maps of maximal rank. Recall that for a complex $X$ one can compute the set $[X, N]$ as follows. If $\operatorname{dim} X<n$, then $\left[X, R P^{n}\right]=H^{1}\left(X ; Z_{2}\right)$; if $\operatorname{dim} X \leqq 2 n$, then $\left[X, C P^{n}\right]=H^{2}(X ; Z)$; if $\operatorname{dim} X \leqq 6$, then $\left[X, Q P^{n}\right]=\left\{u \in H^{4}(X ; Z) \mid \mathrm{Sq}^{2} u=0\right\}$. Here Sq${ }^{2}$ denotes the Steenrod operator of degree 2 (see $[7,8.5 .15]$ ). In each case the correspondence is given by $f \rightarrow f^{*} \iota$, where $f$ denotes a map from $X$ into the projective space, and where $\iota$ denotes generically the fundamental class of the projective space. Thus, we have

$$
\iota \in H^{1}\left(R P^{n} ; Z_{2}\right), \quad \iota \in H^{2}\left(C P^{n} ; Z\right) \quad \text { or } \quad \iota \in H^{4}\left(Q P^{n} ; Z\right)
$$

depending on which of the three projective spaces we are referring to. We call the cohomology class $f^{*} \iota$ the degree of the map $f$. Since the characteristic classes of the projective spaces are known (e.g., see [8]), we now can apply Theorems 1.3 and 1.4 to determine which degrees can occur as the degree of a map of maximal rank from $M$ into a projective space.

As an example we have the following result giving immersions of codimension 2. We assume below that $M$ is a manifold satisfying Condition $(*)$ given in $\$ 1$.

THEOREM 2.1. (a) Let $f: M^{m} \rightarrow R P^{m+2}$ be an orientable map, $3 \leqq m \leqq 9$, with degree $x \in H^{1}\left(M ; Z_{2}\right)$. Then $f$ is homotopic to an immersion if and only if there is a class $v \in H^{2}(M ; Z)$ such that 


$$
\begin{aligned}
& w_{2}(M)+\left(\begin{array}{c}
m+3 \\
2
\end{array}\right) x^{2}=v \quad \bmod 2, \\
& P_{i}(M)+P_{i-1}(M) \cdot v^{2}=0, \quad i=1,2 .
\end{aligned}
$$

(b) Let $f: M^{2 q} \rightarrow C P^{q+1}$ be an orientable map, $2 \leqq q \leqq 4$, with degree $y \in H^{2}(M ; Z)$. Then $f$ is homotopic to an immersion if and only if there is a class $v \in H^{2}(M ; Z)$ such that

$$
\begin{aligned}
w_{2}(M)+q y & =v \bmod 2, \\
P_{i}(M)+P_{i-1}(M) v^{2} & =\left(\begin{array}{c}
q+2 \\
i
\end{array}\right) y^{2}, \quad i=1,2 .
\end{aligned}
$$

(c) Let $f: M^{6} \rightarrow Q P^{2}$ be an orientable map with degree $z \in H^{4}(M ; Z)$. Then $f$ is homotopic to an immersion if and only if there is a class $v \in H^{2}(M ; Z)$ such that $w_{2}(M)=v \bmod 2, P_{1}(M)+v^{2}=2 z$.

Take $M$ to be complex projective space $C P^{m}$. The degree of a map from $C P^{m}$ into $C P^{q}$ or $Q P^{q}$ can now be given simply by an integer taking $\iota \in H^{2}\left(C P^{m} ; Z\right)$ and $\iota^{2} \in H^{4}\left(C P^{m} ; Z\right)$ as generators. Thus by Theorem 2.1 we have

EXAmple 2.2. (a) $C P^{2}$ immerses in $C P^{3}$ only with degrees \pm 1 . (b) $C P^{3}$ immerses in $C P^{4}$ with degree $r$ if and only if there is an integer $s$ such that $5 r^{2}=s^{2}+4$. In particular, there exist immersions with degrees $\pm 1, \pm 5, \pm 13$. (c) $C P^{3}$ immerses in $Q P^{2}$ with degree $r$ if and only if there is an integer $s$ such that $r=2\left(s^{2}+1\right)$.

A result analogous to Theorem 2.1 can be proved for immersions of codimension 1 and for submersions of codimension 1 and 2. We leave the details to the reader.

3. Proof of Theorems $\mathbf{1 . 3}$ and 1.4. We precede the proofs by two simple lemmas.

Lemma 3.1. Let $\xi$ be a vector bundle over a complex $X$ such that $H^{4 i}(X ; Z)$ has no 2-torsion, $i \geqq 0$. Suppose that $w_{1}(\xi)=w_{2}(\xi)=0$ and $P_{i}(\xi)=0, i \geqq 1$. Then $w_{j}(\xi)=0$ for $j \geqq 3$.

Proof. By the formulae of $\mathrm{Wu}[12]$ it suffices to show that $w_{2}(\xi)=0, j \geqq 2$. Consider first $w_{4}(\xi)$. By a theorem of $\mathrm{Wu}$ (cf. [9, Theorem C]), $\theta\left(w_{4}\right)=0$ where $\theta$ denotes the cohomology homomorphism induced by the injection $Z_{2} \subset Z_{4}$. Consequently, $w_{4} \in \beta H^{3}\left(X ; Z_{2}\right)$ where $\beta$ is the Bockstein coboundary associated with the exact sequence $Z_{2} \rightarrow Z_{4} \rightarrow Z_{2}$. But since $H^{4}(X ; Z)$ has no 2-torsion, $\beta H^{3}\left(X ; Z_{2}\right)$ $=0$ and so $w_{4}(\xi)=0$. An inductive argument (using $[9$, Theorem $\mathrm{C}]$ ) now shows that $w_{2^{j}}(\xi)=0$ for all $j \geqq 3$. 
Lemma 3.2. Let $X$ be a complex such that $\operatorname{dim} X \leqq 8, H^{4}(X ; Z)$ has no 2-torsion, and $H^{8}(X ; Z)$ has no 6-torsion. Let $\xi$ and $\eta$ be vector bundles over $X$. Then $(\xi)=(\eta)$ if and only if $w_{i}(\xi)=w_{i}(\eta), i=1,2, P_{i}(\xi)=P_{i}(\eta)$, $i=1,2$.

Proof. By the "product formula" for Stiefel-Whitney classes and Pontryagin classes, it follows that $w_{i}((\xi)-(\eta))=0, P_{i}((\xi)-(\eta))=0$ for $i=1$, 2. (We need here the fact that $H^{4 i}(X ; Z)$ has no 2 -torsion.) Thus by Lemma 3.1, $w_{i}((\xi)-(\eta))=0$ for $i \geqq 1$. The result now follows by applying [10, Theorem 4.3].

Proof of Theorems 1.3 AND 1.4. If $M$ is an open, connected manifold of dimension $m$, then Hirsch [4] shows that $M$ has the homotopy type of an $(m-1)$-complex. Thus if $M$ satisfies Condition $\left({ }^{*}\right)$ in $\$ 1$, it also satisfies the hypotheses of Lemma 3.2. Consequently, Theorems 1.3 and 1.4 now follow by computing the characteristic classes of the bundles in Theorems 1.1 and 1.2 and then applying 3.2. (We need the fact that for $v \in H^{2}(X ; Z), P_{1}(\xi(v))=v^{2}$.) We leave the details to the reader.

Remarks. (a) If $\operatorname{dim} X \leqq 7$, then Lemma 3.2 is given by DoldWhitney [2, Remark, p. 672]. (b) Rather than use [10] to prove Theorems 1.3 and 1.4 , one can study the fibration $\mathrm{BSO}(2) \rightarrow \mathrm{BSO}$. (Note [5].)

\section{REFERENCES}

1. M. Atiyah and F. Hirzebruch, Vector bundles and homogeneous spaces, pp. 7-38, Proc. Sympos. Pure Math., vol. 3, Amer. Math. Soc., Providence, R. I., 1961.

2. A. Dold and $\mathrm{H}$. Whitney, Classification of oriented sphere bundles over a 4-complex, Ann. of Math. 69 (1959), 667-676.

3. M. Hirsch, Immersions of manifolds, Trans. Amer. Math. Soc. 93 (1959), 242276.

4. - On imbedding differentiable manifolds in Euclidean space, Ann. of Math. 73 (1961), 566-571.

5. W. Massey, On the cohomology ring of a sphere bundle, J. Math. Mech. 7 (1958), 265-290.

6. A. Phillips, Submersions of open manifolds, Topology 6 (1966), 171-206.

7. E. Spanier, Algebraic topology, McGraw-Hill, New York, 1966.

8. R. Szczarba, On tangent bundles of fiber spaces and quotient spaces, Amer. J. Math. 86 (1964), 685-697.

9. E. Thomas, On the cohomology of the real Grassmann complexes and the characteristic classes of n-plane bundles, Trans. Amer. Math. Soc. 96 (1960), 67-89.

10. - Homotopy classification of maps by cohomology homomorphisms, Trans. Amer. Math. Soc. 111 (1964), 138-151.

11. - On the existence of immersions and submersions (to appear).

12. W. Wu, Les i-carrés dans une variété grassmannienne, C. R. Acad. Sci. Paris 230 (1950), 918-920.

University of California, Berkeley 